\title{
EXISTENCE OF SOLUTIONS TO BOUNDARY VALUE PROBLEMS AT FULL RESONANCE
}

\section{Kristen Kobylus Abernathy AND Jesús RodRÍGUEZ}

Abstract. The focus of this paper is the study of nonlinear differential equations of the form

$$
\dot{x}_{i}(t)=a_{i}(t) x_{i}(t)+f_{i}\left(\varepsilon, t, x_{1}(t), \cdots, x_{n}(t)\right), \quad i=1,2, \cdots, n,
$$

subject to two-point boundary conditions

$$
b_{i} x_{i}(0)+d_{i} x_{i}(1)=0, \quad i=1,2, \cdots, n .
$$

We formulate sufficient conditions for the existence of solutions based on the dimension of the solution space of the corresponding linear, homogeneous equation and the properties of the nonlinear term when $\varepsilon=0$. We focus on the case when the solution space of the corresponding linear, homogeneous equation is $n$-dimensional; that is, when the system is at full resonance. The argument we use relies on the Lyapunov-Schmidt procedure and the Schauder fixed point theorem.

Mathematics subject classification (2010): 34B08, 34B15.

Keywords and phrases: boundary value problems, Schauder fixed point theorem, Lyapunov-Schmidt procedure, projection.

\section{REFERENCES}

[1] S. BAnCroft, J.K. HALE AND D. SweEt, Alternative problems for nonlinear functional equations, J. Differential Equations, 4 (1968), 40-56.

[2] L. CESARI, Functional analysis and periodic solutions of nonlinear differential equations, Contributions to Differential Equations, 1 (1963), 149-187.

[3] L. CESARI, Functional analysis and Galerkin's method, Michigan Math. J., 11 (1964), 385-414.

[4] S. Chow And J.K. Hale, Methods of Bifurcation Theory, Springer, Berlin, 1982.

[5] D.L. ETheridge AND J. Rodríguez, Periodic solutions of nonlinear discrete-time systems, Appl. Anal., 62 (1996), 119-137.

[6] D.L. ETHERIDGE AND J. RodRíguez, Scalar discrete nonlinear two-point boundary value problems, J. Differ. Equations Appl., 4 (1998), 127-144.

[7] A. Halanay, Solutions periodiques et presque-periodiques des systemes d'equations aux differences finies, Arch. Rational Mech. Anal., 12 (1963), 134-149.

[8] J.K. HALE, Applications of alternative problems, Lecture Notes 71-1, Brown University, Providence, 1971.

[9] J.K. Hale, Ordinary Differential Equations, Second edition, Robert E. Krieger Publishing Co., Inc., Huntington, N.Y., 1980.

[10] D.C. LEWIS, On the role of first integrals in the pertubation of periodic solutions, The Annals of Mathematics, Second Series, 63 (1956), 535-548.

[11] J. RodRÍGUEZ, An alternative method for boundary value problems with large nonlinearities, J. Differential Equations, 43 (1982), 157-167.

[12] J. RodRíGUEZ, Nonlinear differential equations under Stieltjes boundary conditions, Nonlinear Anal., 7 (1983), 107-116.

[13] J. Rodríguez, On resonant discrete boundary value problems, Applicable Anal., 19 (1985), 265274. 
[14] J. RoDRÍGUEZ, Galerkin's method for ordinary differential equations subject to generalized nonlinear boundary conditions, J. Differential Equations, 97 (1992), 112-126.

[15] J. RodrígueZ, Nonlinear discrete Sturm-Liouville problems, J. Math. Anal. Appl., 308 (2005), 380391.

[16] J. RodRíGUEZ AND K. ABERNATHy, On the solvability of nonlinear boundary value problems, Differ. Equ. Appl., 2 (2010), 487-501.

[17] J. RodRíGUEZ AND D. SWeET, Projection methods for nonlinear boundary value problems, J. Differential Equations, 58 (1985), 282-293.

[18] J. RODRÍGUEZ AND P. TAYLOR, Weakly nonlinear discrete multipoint boundary value problems, J. Math. Anal. Appl., 329 (2007), 77-91.

[19] J. RODRíGUEZ AND P. TAYLOR, Scalar discrete nonlinear multipoint boundary value problems, J. Math. Anal. Appl., 330 (2007), 876-890.

[20] J. RODRÍGUEZ AND P. TAYLOR, Multipoint boundary value problems for nonlinear ordinary differential equations, Nonlinear Anal., 68 (2008), 3465-3474.

[21] N. Rouche And J. Mawhin, Ordinary Differential Equations, Surveys Reference Works Math. 5, Advanced Publishing Program, Pitman, Boston MA, 1980. 